\title{
Potential downside issues with telemedicine for individuals with chronic respiratory diseases
}

\author{
Lawrence Paelet ${ }^{1}$, Jonathan Raskin ${ }^{2}$, Richard ZuWallack ${ }^{3,4}$ \\ ${ }^{1}$ Donahue, Durham \& Noonan, P.C. (law firm), Guilford, CT; ${ }^{2}$ Assistant Clinical Professor, Medicine, Pulmonary, \\ Critical Care and Sleep Medicine, Mt Sinai Hospital, New York, NY; ${ }^{3}$ Associate Chief, Pulmonary and Critical Care, \\ Trinity Health of New England, St Francis Hospital, Hartford, CT; ${ }^{4}$ Professor Emeritus, University of Connecticut \\ School of Medicine, Farmington, CT, USA
}

\begin{abstract}
Telemedicine refers to the use of communications technologies to provide or enhance medical care through mitigating the negative effects of patient-caregiver distance on medical evalua-
\end{abstract}

\footnotetext{
Correspondence: Richard ZuWallack, MD, Pulmonary and Critical Care, Trinity Health of New England, St Francis Hospital, 114 Woodland Street, Hartford, CT 06105, USA.

Tel. +1.860.714-4055 - Fax: +1.860.714-8035.

E-mail: rlzuwallack@gmail.com
}

Key words: telemedicine; telehealth; chronic respiratory disease; privacy, confidentiality.

Acknowledgements (dedication): The authors dedicate this paper to the memory of Dr. Claudio F. Donner, a friend, colleague and a source of inspiration to respirologists around the world.

Contributions: LP, conceptualization and writing of paper; JR, writing of paper; RZW, conceptualization, writing first draft, writing of paper. All the authors have read and approved the final version of the manuscript and agreed to be accountable for all aspects of the work.

Conflict of interest: The authors declare that they have no competing interests, and all authors confirm accuracy.

Funding: There was no external funding for this endeavor.

Ethics approval: Since this was a review paper and human subjects were not studied or reported on, Institutional Review Board approval was not needed.

Received for publication: 21 October 2021

Accepted for publication: 21 Janaury 2022.

Publisher's note: All claims expressed in this article are solely those of the authors and do not necessarily represent those of their affiliated organizations, or those of the publisher, the editors and the reviewers. Any product that may be evaluated in this article or claim that may be made by its manufacturer is not guaranteed or endorsed by the publisher.

${ }^{\circ}$ Copyright: the Author(s), 2022

Licensee PAGEPress, Italy

Monaldi Archives for Chest Disease 2022; $92: 2132$

doi: 10.4081 /monaldi.2022.2132

This article is distributed under the terms of the Creative Commons Attribution-NonCommercial International License (CC BY-NC 4.0) which permits any noncommercial use, distribution, and reproduction in any medium, provided the original author(s) and source are credited. tion and treatment. The general concept, telemedicine, can refer to a number of interventions, such as telemonitoring, tele-consultations, tele-education, tele-communication, and tele-rehabilitation. While telemedicine has seen steady growth, its trajectory has increased during the COVID-19 pandemic. As a tool in health care delivery, telemedicine is often met with patient satisfaction often resulting from ease of use and accessibility. Additionally, outcomes may improve, although the medical literature is not consistent in this regard. However, enthusiasm over its beneficial effects should be tempered by negative aspects, including the decrease in direct patient-clinician interaction (such as loss of information from the physical examination) and potentially serious privacy risks. Finding a happy medium between positive and negative features of telemedicine remains a work in progress.

\section{Telemedicine: concepts}

Telemedicine, telehealth, and 'connected health' [1] are almost identical concepts that refer to the use of communications technologies as tools to provide or enhance medical care, typically through reducing or eliminating the negative effects of distance between the patient and care-giver on health care delivery. Telehealth differs slightly from telemedicine in that it (telehealth) emphasizes data analytics in strategizing health care [2]. "Connected health" is similar in concept to telemedicine, although it emphasizes the integration of patient data (aided by technology) across multiple providers and settings [2], thereby emphasizing the increased availability of data (integration) to multiple entities and providers over the tool (telemedicine technologies). This is in accord with the worldwide greater emphasis on integration of care as a means to improve outcomes [3]. Since these terms do not differ substantially in meaning and there is overlap in usage, for the purpose of consistency in this discussion we will use the term, "telemedicine". Through improving lines of communication, often bi-directionally, telemedicine has potential to improve efficiency of delivery, quality of care, or availability of services.

The World Health Organization defined telemedicine in 2010 as "The delivery of health care services, where distance is a critical factor, by all health care professionals using information and communication technologies for the exchange of valid information for diagnosis, treatment and prevention of disease and injuries, research and evaluation, and for the continuing education of health care providers, all in the interests of advancing the health of individuals and their communities" [4]. A more recent definition (2017) from the US Health Resources and Services 
Administration is: "the use of electronic information and telecommunications technologies to support long-distance clinical health care, patient and professional health-related education, public health and health administration. Technologies include videoconferencing, the Internet, store-and-forward imaging, streaming media, and terrestrial and wireless communications" [5]. Synchronous, live video interaction between the patient and health provider (virtual visit) is a prime example of telemedicine [6]. Telehealth may also involve bi-directional transfer of sensitive health data among health care providers and patients [7]. Finally, another form of telemedicine is the asynchronous, stored transfer of information between health care providers and patients [6].

While the concept of telemedicine is relatively straightforward, its applications in medical treatment vary considerably, making assessments of its general effectiveness problematic, as reflected in inconsistent results in clinical trials (8-15). While providing medical services at a distance is common to almost all, considerable variation in approaches reflects differing needs in distinct clinical settings. For example, telemedicine can be used to provide greater access to medical care in rural areas; to those who cannot reasonably make in-person health care visits, such as those who are functionally- or cognitively- impaired or those with inadequate support systems; or to help reduce disparities in medical services in medically-underserved areas.

Telemedicine interventions can also be used to promote bidirectional transfer of important clinical information to monitor and adjust therapy for those patients with unstable clinical situations, such as those highly-vulnerable to adverse outcome in the days to weeks following discharge for a COPD exacerbation. Examples of telemedicine approaches in this setting include bidirectional electronic transfer of information: i) symptom scores and physiologic data from the patient to the health care provider; and ii) changes in medical therapy sent from the provider to the patient based on interpretation of this information [3].

\section{Telemedicine as a tool to enhance care for the respiratory patient}

The potential benefits of telemedicine listed above should be applicable to the patient with chronic respiratory disease, who is often limited by the burdens imposed by the disease, its common comorbidities, and the (often) multiple therapies required to address them [16]. Table 1 lists several forms of telemedicine that could be used for the patient with chronic respiratory disease.

A recent review of telemedicine for patients with chronic obstructive pulmonary disease (COPD), including 56 articles, placed interventions into four general categories: i) Tele-rehabilitation; ii) Tele-health education and self-management training; iii) Tele-monitoring; and iv) Mobile applications and websites [17]. While conflicting results were present and the heterogeneity of interventions made inferences problematic, the authors noted a positive tendency in non-inferiority studies toward benefit from tele-rehabilitation, tele-education and self-management, early detection of exacerbations, psychosocial support and smoking cessation. Advantages in traditional health care utilization outcomes compared to conventional COPD management could not be established in most studies, although increased convenience and availability of telemedicine are of obvious importance to patients.

Another recent review of telemedicine in COPD of 19 studies involving 301 patients focused on qualitative outcomes [18]. Positive outcomes included perceived ease of use, easy access to health services, usefulness, improvement or supplementation of standard care, sense of security and encouragement, relationships with healthcare professionals, reduced use of health care services through early detection of changes in patients' conditions, and development of positive attitudes toward healthy behaviors such as exercise.

Pulmonary rehabilitation, which is typically center-based and includes components of goal setting, exercise training and self-management training, is increasingly supported with remote technologies. A relatively large survey of people participating in traditional pulmonary rehabilitation demonstrated that the patients already had substantial technology engagement and good self-reported technology competence. Furthermore, many were willing to use telerehabilitation, especially those who were regular users of technology devices [19].

\section{Implementation of telemedicine in the era of COVID-19}

The proverb, "necessity is the mother of invention" explains, in part, the striking increase in use of telemedicine services during the

\section{Table 1. Forms of telemedicine for the respiratory patient.}

- Telemonitoring: unidirectional or bidirectional clinical information transfer, such as oxygen saturation via pulse oximetry, daily weights, dyspnea scores [40]

- Health care provider visits, including with primary care providers and consultants when in-person visits are not feasible, due to safety concerns (COVID-19 pandemic), functional or social limitations, distance to the provider [41]

- Tele-education: such as providing didactic sessions or enhancing collaborative self management interventions, including respiratory exacerbation action plans [42]

- Tele-communication: to enhance lines of communication between health care provider and patients or families, thereby promoting integrated care for the respiratory patient [43]

- Tele-rehabilitation: augmenting or providing pulmonary rehabilitation services, typically through web-based programs $[14,19,29,42,44,45]$

- Helping manage patients residing at home with chronic respiratory failure needing ventilator support [46-48]

- Feedback programs to promote general health; for these to qualify as telemedicine they must work over a network [7], such as web-based programs providing step counts and positive reinforcement to promote regular activity in sedentary individuals [49]

- The ability of patients to conveniently view their medical problem list, medications, laboratory and tests results, and scheduled appointments on the internet [2]

- Home-based support group video conferencing led by a health professional [50] 
2020-2021 COVID-19 pandemic [20] - when one-on-one encounters between vulnerable patients and health care providers were potentially hazardous [21]. This increase in use of telemedicine was experienced globally [22], and was especially so for individuals at high risk for adverse outcome [20,23], including those with COPD [24]), or for health care systems stretched to or beyond their limits during the pandemic. Adding to this, some health care systems put telemedicine on an equal or near-equal footing to in-person visits for financial reimbursement to health care providers during the pandemic $[25,26]$.

Despite the above inducements, the increase in telemedicine encounters did not match the decrease in standard office visits, indicating a drop-off in overall medical care over this period. For example, in the United States, Quarter 22019 (pre-pandemic) total office visits decreased from approximately 122 million to 59 million compared with the same quarter in 2020 (during the pandemic), while telemedicine encounters increased from approximately 1.6 million to 35 million over these two periods [27]. Furthermore, demographic and socioeconomic disparities in the use of telemedicine for medical care during the pandemic in the United States have been uncovered, with males, older individuals, and those with lower median household income less likely to complete a virtual visit [28].

\section{Risks inherent to the use of telemedicine}

\section{Quality of care risks}

The metaphor, "double-edged sword", can be applied to telemedicine as a tool in the management of the patient with chronic disease such as COPD. As mentioned earlier, obvious benefits include the near-simultaneous transfer of important and objective clinical data in situations that would preclude direct, personal interactions. An example is monitoring and feedback to the patient in the immediate post-hospitalization period after a COPD exacerbation, when clinical stability has not yet been achieved and the patient is at high risk for untoward events including re-hospitalization or death. Another benefit is the convenience (and possible increased safety in the COVID-19 pandemic era) of virtual patient - health care provider encounters to provide ongoing medical care. These potential benefits come with some inherent risks:

1) Reliance on telemedicine as a sole intervention could be problematic should the equipment or system fail [29]. This would be of obvious importance in ongoing monitoring of critical variables, such as oxygen saturation or ventilator use in individuals with respiratory failure;

2) The virtual encounter (such as tele-consultation) does not transmit certain, important information, such as what would be gleaned from a face-to-face physical examination;

3) The absence of direct human contact, even with video input, precludes the transmission of some nuances of the patient healthcare provider interaction, such as facial or bodily signs resulting from the verbal interaction. As a result of this, the telemedicine encounter may not meet traditional standards of care. To meet this deficiency, it has been suggested that clinicians use nonverbal communication strategies with limited video capabilities, such as sitting up and leaning forward, using heightened facial expressions and head gesturing, and purposely trying to maintain eye contact via the web camera [23]. Training videos have been created to help meet this challenge [30]; and

4) Due to the inability to conduct an in-person exam, the physician may be required to rely on the observations documented in the past by other providers, exposing the patient to the risks associated with inconsistencies or variations among observers.

From the above list, arguably the major limitation of virtual care is the reduction in objective information from the physical examination that would have come from a direct patient-clinician interaction. This deficiency of information could impair the ability to establish a proper diagnosis and treatment plan [23]. This interaction from afar can place the clinician in unchartered waters of decision-making with less-than-optimal knowledge of clinical status. The reduction in information transfer goes in both directions: the patient may miss subtle cues from the clinician's demeanor while explaining the medical problem. Thus, the decision on whether or not to utilize telemedicine rests on the balance between enhanced utilization and satisfaction and potentially-reduced clinical information. Whether this reduction in information transfer is counterbalanced by the convenience, accessibility, and efficiency of the virtual visit remains to be determined.

An example of a potential issue with telemedicine for the respiratory patient is the clinician's decision on whether or not to initiate oral steroid and/or antibiotic therapy for a COPD patient with an exacerbation. The COPD exacerbation causes severe and prolonged increases in symptoms and functional deterioration; it also results in high subsequent health care utilization and mortality risk [31-33]. A virtual interaction might lead the clinician to miss the severity of the acute illness that an in-person assessment could otherwise provide. In this potentially high-stakes situation, not having information from a direct interaction and physical examination, with resultant over-reliance on historical data from our patients is not without risk.

\section{Privacy and security risks}

The terms, "confidentiality", "privacy" and "security" as applied to telehealth are sometimes used interchangeably, although they differ in concept. For the purpose of this paper we define confidentiality as the obligation a trusted individual to safeguard another person's personal data from being released. Privacy is a person's right to have his or her personal data safeguarded from other parties [34]. Privacy is a right of protection against an intrusion upon an individual's seclusion or solitude or private affairs, or public disclosure of embarrassing private facts about an individual. Security refers to the process required to implement these safeguards [35]. As applied to telemedicine, security is primarily cybersecurity.

Cybersecurity risks are not limited to telemedicine, as health data are increasingly transmitted and stored in the Internet Cloud [36]; however, high on the list of risks is privacy loss. As a result, protected health information is regulated in the United States and other countries. Privacy risks involve the potential loss of control of the collection, use or disclosure of protected health information [7]. An example of this type of breech with respect to telemedicine is the inadvertent transmission of potentially sensitive ongoing symptom scores, physiologic variables, etc., to persons or entities other than their intended recipients. Protected health information (PHI) and personally identifiable information (PII) can command a high price in the black market [6].

In the United States, attempting to meet patient privacy regulations laid out in the Health Insurance Portability and Accountability Act (HIPAA) of $1996^{1}$ has led to a barrier to implementation of telemedicine [26], reflecting the particular vulnera-

Health Insurance Portability and Accountabiity Act of 1996, 1996 Enacted H.R. 3103, 104 Enacted H.R. 3103, 110 Stat. 1936, 104 P.L. 191, 1996 Enacted H.R. 3103, 104 Enacted H.R. 3103. 
bility of electronically-transmitted protected information. HIPAA has a Privacy Rule (establishing standards for PHI), ${ }^{2}$ a Security Rule (a set of security standards to protect health information stored or transmitted electronically), ${ }^{3}$ and a Breach Notification

2 The HIPAA privacy rule is a set of federal regulations that regulate the use and disclosure of protected health information by covered entities and business associates. 45 C.F.R. $\S 164.500-.534$ (codifying the HIPAA Privacy Rule). Protected health information ("PHI") is defined as "individually identifiable health information ... that is ... [t]ransmitted by electronic media [,] ... [m]aintained in electronic media [,] or ... [t]ransmitted or maintained in any other form or medium.” Id. $\S 160.103$. The HIPAA Privacy Rule requires covered entities and business associates to establish technical "safeguards to protect the privacy of [PHI]" and to "reasonably safeguard [PHI] from ... intentional [and] unintentional use[s] [and] disclosure[s] that ... violat[e]" the HIPAA Privacy Rule. Id. § 164.530(c)(1)-(2).

3 The HIPAA Security Rule is a set of federal regulations that requires covered entities and business associates to: "(1) [e]nsure the confidentiality, integrity, and availability of all [ePHI] the covered entity or business associate creates, receives, maintains, or transmits," and "(2) [p]rotect against ... reasonably anticipated threats or hazards to the security or integrity of such [ePHI]." See 45 C.F.R. § 164.306(a)(1)-(2); see also id $\S \S$ 164.302-.318 (codifying the HIPAA Security Rule).

4 The HIPAA Breach Notification Rule requires covered entities to notify individuals, the media, and the Secretary of the U.S. Department of Health and Human Services ("HHS") in specified situations in which a breach of unsecured PHI has been discovered. See 45 C.F.R. $\S \S 164.400$ .414 (codifying the Breach Notification Rule).

5 In March, 2020, the OCR announced that, effective immediately, it was exercising its enforcement discretion to not impose penalties for HIPAA violations against healthcare providers in connection with their good faith provision of telehealth using communication technologies during the COVID-19 nationwide public health emergency. A copy of this notification is set forth at the following website: https://www.hhs.gov/hipaa/for-professionals/specialtopics/emergency-preparedness/notification-enforcement-discretion-telehealth/index.html The Notification was accompanied by information in the form of a set of "Frequently Asked Questions" about telehealth remote communications, a copy of which is available at the following website: https://www.hhs.gov/sites/default/files/telehealth-faqs-508.pdf - PDF
Rule (requiring covered entities to report breaches) ${ }^{4}[6,43]$.

The regulations under the HIPAA legislation specifically pertain to health care providers who bill electronically, health plans, and health care clearinghouses, with its security rules establishing technical, physical and administrative safeguards, including encryption of data [7]. These regulated entities are relatively narrow in scope. For example, telemedicine apps that collect data directly from the patient are not covered entities under HIPAA, setting the stage for transmission of potentially sensitive data outside the purview and regulation of HIPPA [37]. Additionally, health care providers' use of online video and chat platforms such as Facebook to consult with patients is not regulated by HIPAA. Adding to potential privacy issues, in order to meet the sudden increased rationale and need for telemedicine services during the COVID-19 pandemic, an administrative decision was made by the Office of Civil Rights ("OCR") at the U.S. Department of Health and Human Services ("HHS") to forego enforcement of HIPAA violations for those infractions that occurred in good faith. One example of the relaxed enforcement is the temporary elimination of the encryption requirement for transfer of telemedicine information $^{5}$ [37]. Providers were encouraged to notify patients that thirdparty applications might potentially introduce privacy risks, and they were told that they were authorized to use certain non-public facing applications that allowed for video chats, but that they should avoid the use of public-facing communication applications for telehealth visits.

Table 2 lists twelve requirements for telemedicine services to maximize security and privacy by users [38].

\section{Conclusions}

Telemedicine, encompassing communications technologies to provide or enhance medical care across distances, is a promising tool in facilitating and augmenting medical care. For the individual with chronic respiratory disease such as COPD, its potential

Table 2. Requirements for telemedicine services to maximize security and privacy by users. Adapted from Vega-Barbas M, Seoane F, Pau I. Int J Environ Res Public Health 2019;16:693 [38].

1. Existing security conditions must not alter the general, positive aim of telehealth to enhance health care services

2. The system must ensure custody over all electronically-stored information and must adapt to accumulation of data over time, providing optimal access to enhance integration of care

3. Certain data may need to be disclosed to other entities, per legally-mandated regulations; this release of information must be tracked

4. Communication between the patient and health care provider must follow the following criteria: confidentiality, authentication, non-repudiation, and integrity

5. Patient data made anonymous through eliminating identifiers can be used for research purposes, under the direction of an ethics committee or similar entity, such as an Institutional Review Board

6. Sharing patient data with other health care providers must be regulated and subject to audit

7. In general, patients (as owners of the data) have the right to possession of their clinical information and may authorize the processing of this information for a specific purpose, providing proper safeguards are in place

8. Patients must have the right to eliminate all information specifically related to them

9. Patients must be aware of what is occurring in their interactions with health care providers and must be able to expressly confirm the data exchange, and acknowledge the privacy risks associated with video chat applications

10. Security hardware must be used as a basis for identification of the patient

11. In some jurisdictions, secure terminals must be used for the generation of electronic signatures

12. The security measures that are implemented must enable mechanisms of personalization that enhance the perception of security by the user

13. The use of public-facing communication applications should be avoided for telehealth visits. 
uses include bidirectional clinical information transfer, tele-visits and tele-consultation, facilitation of education, tele-communication, tele-rehabilitation, and aiding the management of patients requiring ventilator support. These applications are in early stages of development and implementation. The decision to utilize these tools must be made on an individual basis, balancing benefits and risks, taking into consideration those measures necessary to mitigate those potential risks.

Over and above the balance between efficacy and safety, the use of this new technology may itself be a means of changing clinical perspectives, insights and thought processes. As the delivery of a service is changed so are the parameters of care. Marshall McLuhan's insight that the medium is the message may well be relevant as we test these new approaches to patient care. "A new medium is never an addition to an old one, nor does it leave the old one in peace. It never ceases to oppress the old media until it finds new shapes and positions for them" [39]. We must proceed with eyes wide open to risks and benefits as we engage with novel telemedicine technology while creating mechanisms to patient safety. While our responsibilities to patient care have not changed, the landscape of clinical medicine is changing continually. As such, we must ensure that innovation is complementary to our overarching commitment to excellence in patient care.

\section{References}

1. Hale TM, Kvedar JC. Privacy and security concerns in telehealth. Virtual Mentor 2014;16:981-5.

2. Kuziemsky CE, Gogia SB, Househ M, et al. Balancing health information exchange and privacy governance from a patientcentred connected health and telehealth perspective. Yearb Med inform 2018;27:48-54.

3. Donner CF, Raskin J, ZuWallack R, et al. Incorporating telemedicine into the integrated care of the copd patient a summary of an interdisciplinary workshop held in stresa, Italy, 7-8 september 2017. Respir Med 2018;143:91-102.

4. World Health Organization. Telemedicine: Opportunities and developments in member states. 2010. [cited 2021]. Available from: https://www.who.int/goe/publications/goe_telemedicine_2010.pdf

5. Watzlaf VJM, Zhou L, Dealmeida DR, Hartman LM. A systematic review of research studies examining telehealth privacy and security practices used by healthcare providers. Int J Telerehabil 2017;9:39-59.

6. Healthcare \& Public Health Sector Coordinating Councils. Health industry cybersecurity - securing telehealth and telemedicine. 2021. Available from: https://www.aha.org/system/files/media/file/2021/04/health-industry-cybersecuritysecuring-telehealth-and-telemedicin-april-2021.pdf

7. Hall JL, McGraw D. For telehealth to succeed, privacy and security risks must be identified and addressed. Health Aff (Millwood) 2014;33:216-21.

8. Kruse C, Pesek B, Anderson MR, et al. Telemonitoring to manage chronic obstructive pulmonary disease: Systematic literature review. JMIR Med Inform 2019;7:e11496.

9. McCabe C, McCann M, Brady AM. Computer and mobile technology interventions for self-management in chronic obstructive pulmonary disease. Cochrane Database Syst Rev 2017;5:CD011425.

10. Sul AR, Lyu DH, Park DA. Effectiveness of telemonitoring versus usual care for chronic obstructive pulmonary disease: A systematic review and meta-analysis. J Telemed Telecare 2020;26:189-99.

11. Soriano JB, Garcia-Rio F, Vazquez-Espinosa E, et al. A multicentre, randomized controlled trial of telehealth for the management of copd. Respir Med 2018;144:74-81.

12. Lundell S, Holmner A, Rehn B, et al. Telehealthcare in copd: A systematic review and meta-analysis on physical outcomes and dyspnea. Respir Med 2015;109:11-26.

13. Cox NS, Dal Corso S, Hansen H, et al. Telerehabilitation for chronic respiratory disease. Cochrane Database Syst Rev 2021;1:CD013040.

14. Vasilopoulou M, Papaioannou AI, Kaltsakas G, et al. Homebased maintenance tele-rehabilitation reduces the risk for acute exacerbations of COPD, hospitalisations and emergency department visits. Eur Respir J 2017;49: 1602129.

15. Duiverman ML, Vonk JM, Bladder G, et al. Home initiation of chronic non-invasive ventilation in copd patients with chronic hypercapnic respiratory failure: A randomised controlled trial. Thorax 2020;75:244-52.

16. Vitacca M, Montini A, Comini L. How will telemedicine change clinical practice in chronic obstructive pulmonary disease? Ther Adv Respir Dis 2018;12:1753465818754778.

17. Barbosa MT, Sousa CS, Morais-Almeida M, et al. Telemedicine in COPD: An overview by topics. COPD 2020;17:601-17.

18. Li W, Liu W, Liu S, et al. Perceptions of patients with chronic obstructive pulmonary disease towards telemedicine: A qualitative systematic review. Heart Lung 2021;50:675-84.

19. Seidman Z, McNamara R, Wootton S, et al. People attending pulmonary rehabilitation demonstrate a substantial engagement with technology and willingness to use telerehabilitation: A survey. J Physiother 2017;63:175-81.

20. Hincapie MA, Gallego JC, Gempeler A, et al. Implementation and usefulness of telemedicine during the covid-19 pandemic: A scoping review. J Prim Care Community Health 2020;11:2150132720980612.

21. Kaplan B. Revisiting health information technology ethical, legal, and social issues and evaluation: Telehealth/telemedicine and Covid-19. Int J Med Inform 2020;143:104239.

22. Bhaskar S, Bradley S, Chattu VK, et al. Telemedicine across the globe-position paper from the covid-19 pandemic health system resilience program (reprogram) international consortium (part 1). Front Public Health 2020;8:556720.

23. Zulman DM, Verghese A. Virtual care, telemedicine visits, and real connection in the era of covid-19: Unforeseen opportunity in the face of adversity. JAMA 2021;325:437-8.

24. Boyce DM, Thomashow BM, Sullivan J, Tal-Singer R. New adopters of telemedicine during the Coronavirus-19 pandemic in respondents to an online community survey: The case for access to remote management tools for individuals with chronic obstructive pulmonary disease. Chronic Obstr Pulm Dis 2021;8:213-8.

25. Patel SY, Mehrotra A, Huskamp HA, et al. Trends in outpatient care delivery and telemedicine during the covid-19 pandemic in the US. JAMA Inter Med 2021;181:388-91.

26. Shachar C, Engel J, Elwyn G. Implications for telehealth in a postpandemic future: Regulatory and privacy issues. JAMA 2020;323:2375-6.

27. Alexander GC, Tajanlangit M, Heyward J, et al. Use and content of primary care office-based vs telemedicine care visits during the covid-19 pandemic in the us. JAMA Netw Open 2020;3:e2021476.

28. Darrat I, Tam S, Boulis M, Williams AM. Socioeconomic dis- 
parities in patient use of telehealth during the coronavirus disease 2019 surge. J JAMA Otolaryngol Head Neck Surg 2021;147:287-95.

29. Ambrosino N, Vagheggini G, Mazzoleni S, Vitacca M. Telemedicine in chronic obstructive pulmonary disease. Breathe 2016;12:350-6.

30. Academy of Communication in Healthcare [Internet]. Telehealth communication: Quick tips. 6 July 2021. Available from: http://www.achonline.org/telehealth

31. Cote CG, Dordelly LJ, Celli BR. Impact of copd exacerbations on patient-centered outcomes. Chest 2007;131:696-704.

32. Esteban C, Castro-Acosta A, Alvarez-Martinez CJ, et al. Predictors of one-year mortality after hospitalization for an exacerbation of copd. BMC Pulm Med 2018;18:18.

33. Slenter RH, Sprooten RT, Kotz D, et al. Predictors of 1-year mortality at hospital admission for acute exacerbations of chronic obstructive pulmonary disease. Respiration 2013;85:15-26.

34. Techopedia [Internet]. What is the difference between privacy, confidentiality and security? 2021. Available from: https://www.techopedia.com/7/29803/security/what-is-the-difference-between-privacy-confidentiality-and-security

35. Brumen B, Hericko M, Sevcnikar A, et al. Outsourcing medical data analyses: Can technology overcome legal, privacy, and confidentiality issues? J Med Internet Res 2013;15:e283.

36. Gerdes Martin FR. End-to-end security and privacy protection for co-operative access to heath and care data in a telehealth trial system for remote supervision of COPD-patients. Proceedings 13th Scandinavian Conference on Health Informatics, Tromsø, Norway.

37. Bassan S. Data privacy considerations for telehealth consumers amid Covid-19. J Law th Biosci 2020;7:1saa075.

38. Vega-Barbas M, Seoane F, Pau I. Characterization of user-centered security in telehealth services. Int J Environ Res Public Health 2019;16:693.

39. McLuhan M, Gordon WT. Understanding media: The extensions of man. Corte Madera: Gingko Press; 2003.
40. Fried TR. Shared decision making--finding the sweet spot. N Engl J Med 2016;374:104-6.

41. Persaud YK, Portnoy JM. Ten rules for implementation of a telemedicine program to care for patients with asthma. J Allergy Clin Immunol Pract 2021;9:13-21.

42. Chaplin E, Hewitt S, Apps L, et al. The evaluation of an interactive web-based pulmonary rehabilitation programme: Protocol for the web space for copd feasibility study. BMJ Open 2015;5:e008055.

43. Stewart JI, Moyle S, Criner GJ, et al. Automated telecommunication to obtain longitudinal follow-up in a multicenter cross-sectional copd study. COPD 2012;9:466-72.

44. Selzler AM, Wald J, Sedeno M, et al. Telehealth pulmonary rehabilitation: A review of the literature and an example of a nationwide initiative to improve the accessibility of pulmonary rehabilitation. Chron Respir Dis 2018;15:41-7.

45. Cox NS, McDonald CF, Alison JA, et al. Telerehabilitation versus traditional centre-based pulmonary rehabilitation for people with chronic respiratory disease: Protocol for a randomised controlled trial. BMC Pulm Med 2018;18:71.

46. Masefield S, Vitacca M, Dreher M, et al. Attitudes and preferences of home mechanical ventilation users from four european countries: An ERS/ELF survey. ERJ Open Res 2017;3:00015-2017.

47. Crimi C, Noto A, Princi P, et al. Domiciliary non-invasive ventilation in copd: An international survey of indications and practices. COPD 2016;13:483-90.

48. Soo CI, Chan Y, Loh EC, Pang YK. Telehealth: "Virtual" lifeline for home-ventilated patients during the covid-19 pandemic. ERJ Open Res 2020;6:00399-2020.

49. Mantoani LC, Rubio N, McKinstry B, et al. Interventions to modify physical activity in patients with COPD: A systematic review. Eur Respir J 2016;48:69-81.

50. Banbury A, Nancarrow S, Dart J, et al.Telehealth interventions delivering home-based support group videoconferencing: Systematic review. J Med Internet Res 2018;20:e25. 